IV. FAR ULTRA-VIOLET RADIATION : STARS AND GALAXIES 


\title{
FAR ULTRA-VIOLET RADIATION : STARS, NEBULAE AND INTERSTELLAR MATTER
}

\author{
by M. J. Seaton \\ (University College, London, United Kingdom)
}

RÉsumé. - Le flux ultraviolet observé dans les étoiles chaudes est inférieur au flux calculé. Les causes possibles de cette différence sont discutées : absorption interstellaire, écarts à l'ETL, absorption moléculaire, absorption ato. mique, effet de blanketting. L'observation des nébuleuses planétaires permet d'étudier le rayonnement $U$. V. des étoiles très chaudes.

ABstract. - The ultra-violet flux radiated by hot stars is found to be smaller than the value deduced from theory. The possible origins of this discrepancy are discussed : interstellar absorption, deviation from local thermodynamic equilibrium, molecular absorption, blanketting effect. Observations of planetary nebulae provide data about UV radiation from very hot stars.

Резюме. - Наблюдаемый ультрафиолетовый поток горячих звезд меньше вычисленного потока. Обсуждены возможеные причины этой разницы : межзвездное поглощение, отклонения в локальном термодинамическом равновесии, молеклярное поглищение, атомное поглощение эффект blankett.ng. Наблюдение планетарных туманностей позволяет изучать излучение UV горячих звезд.

\section{ROCKET OBSERVATIONS OF STELLAR ULTRA-VIOLET.}

Spectral scans using a dispersive system have been made by STECHER and MilligaN [1] for $\lambda \geqslant 1600 \AA$ and observations using filters with transmission at effective wavelengths $\lambda_{0}$ have been made by CHUBB and BYRAM [2] for $\lambda_{0}=1314$ and $1427 \AA$, by Alexander, Bowen and HedDLE [3] for $\lambda_{0}=1950 \AA$ and by GULLEDGE and PAOKER [4], [5] for $\lambda_{0}=2120 \AA$. The results obtained are conveniently expressed as fluxes $F_{\lambda}$ above the earth's atmosphere, in units of $10^{-9} \mathrm{ergs} \mathrm{cm}^{-2} \mathrm{~s}^{-1} \AA^{-1}$.

Figure 1 shows results for stars for which the spectral scans can be compared with the filter measurements. There is seen to be fair agreement in the general flux levels but more observations are clearly needed in order to be at all certain about the precise spectral distributions. In particular there remains some doubt about the reality of the sharp intensity drop at $\sim 2400 \AA$ obtained by Stecher and Mituigar.

Most of the observations are for B stars, stars hotter than B 0 being rare and stars cooler than A 0 being rather faint at $U$. V. wavelengths.
From the standpoint of theory the $B$ stars are of interest because the maximum of the Planck function comes in a region where the opacity is small and varies rapidly with frequency; of all stars, B stars are the most " non-grey".

With the exception of the F 0 star $\alpha$ Carinae, for which the observations [1], [3] are in satisfactory agreement with model atmosphere calculations by Canavageia and Pecker [6], all of the observed U. V. fluxes are significantly smaller than those predicted by theory. This has been discussed in a number of papers [5], [7], [14].

The basic assumptions made in calculating models are that stellar atmospheres are in radiative equilibrium, hydrostatic equilibrium and local thermodynamic equilibrium. In addition it is assumed that the main sources of opacity for hot stars are electron scattering and continuous absorption by $\mathrm{H}, \mathrm{He}$ and $\mathrm{H}^{-}$. We first discuss the results obtained on making these assumptions. The mathematical problems of model construction are of some complexity but accurate solutions can be obtained using large computers. Greatly improved calculations have been obtained in the past two or three years, as a result both of the stimulation provided by the rocket results and of the technical advances in machine computation. The basic parameters are the effective tempera- 

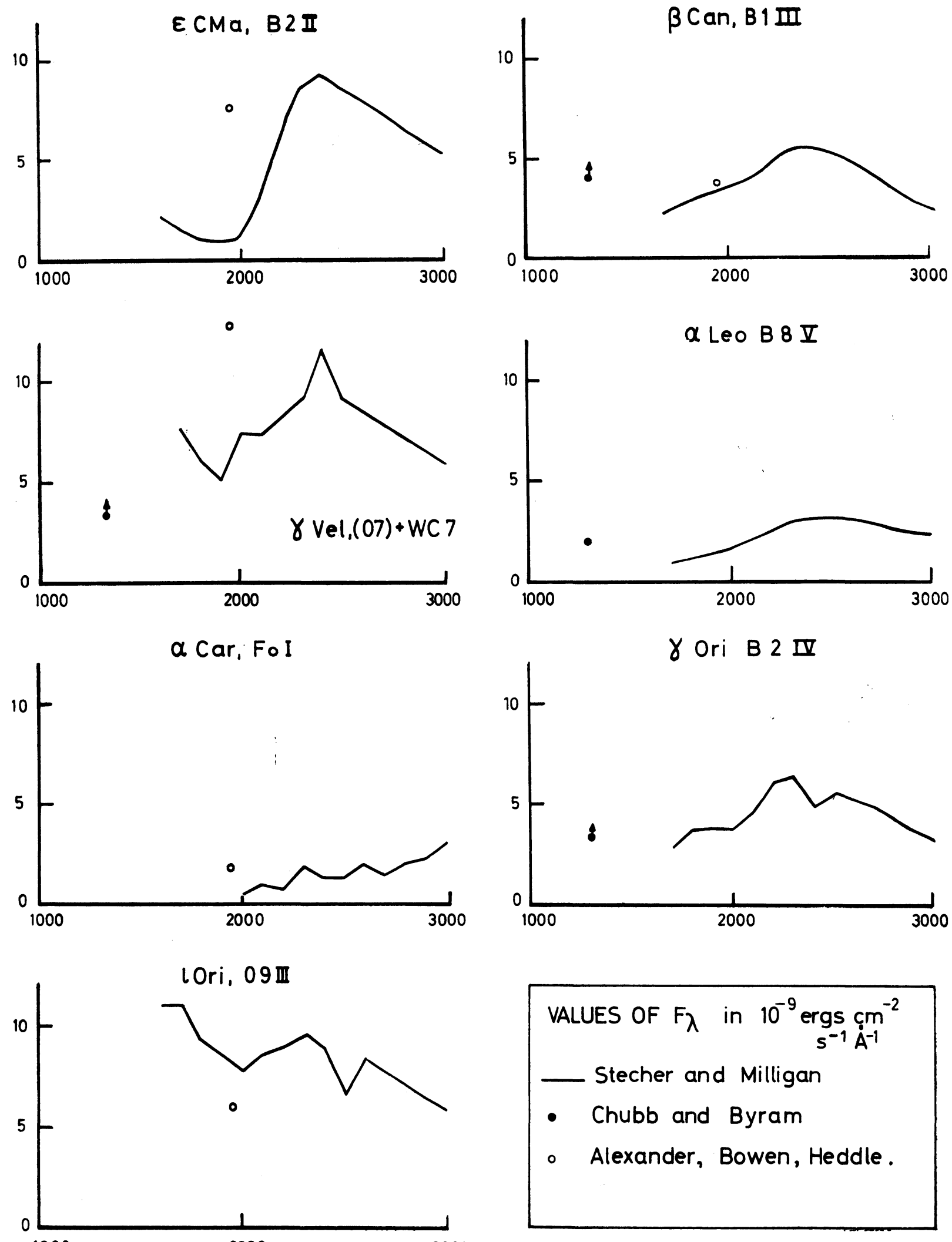

- Observations of stellar $u-v, \quad F_{\lambda}$ in $10-9$ ergs $\mathrm{cm}^{-2} \mathrm{~s}^{-1} \AA_{-1}^{-1}$

Results from spectral scans [1] are compared with results obtained using filters $[2,3]$, 


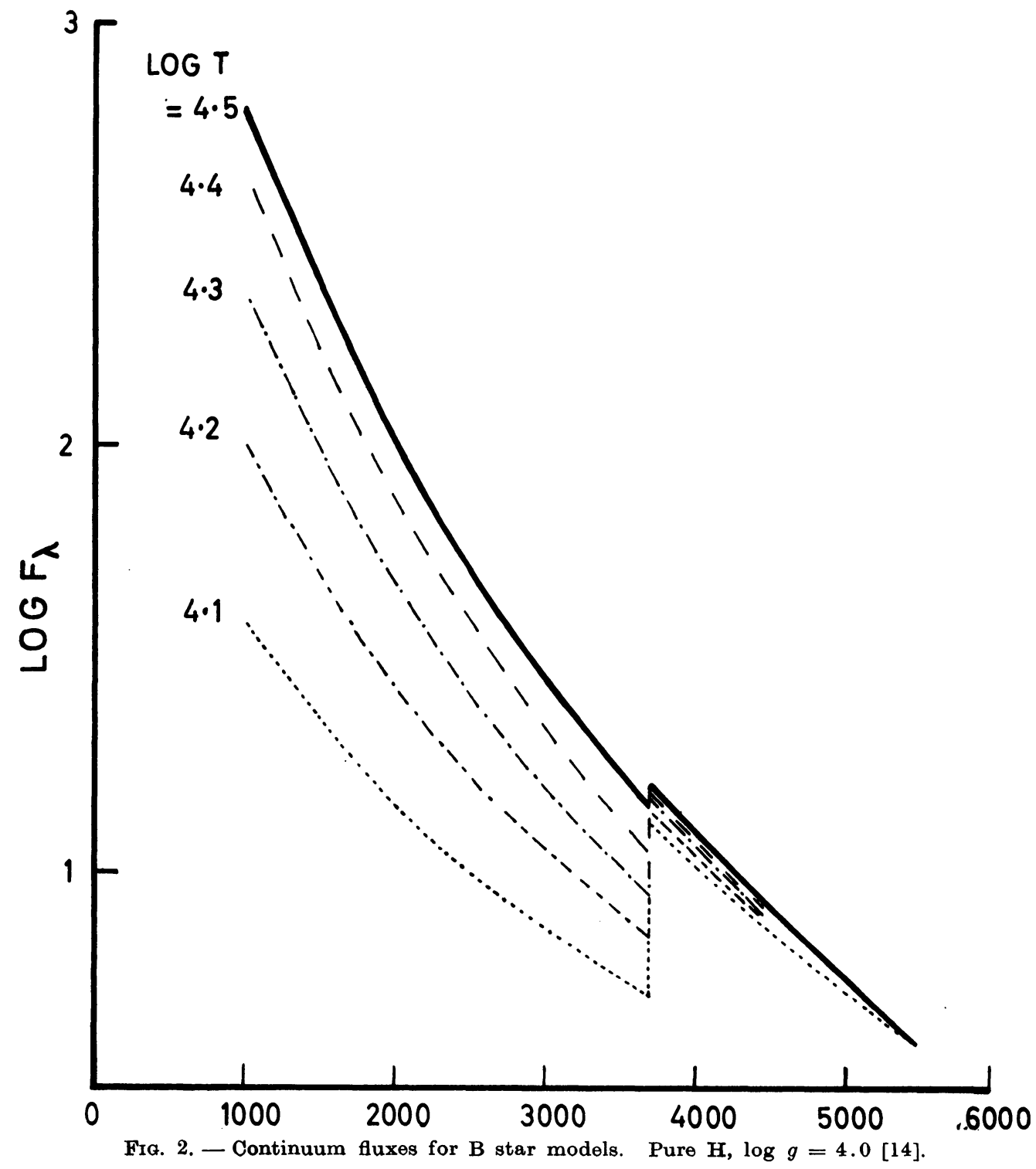

ture $\mathrm{T}_{e}$, the surface gravity $g$ and the chemical composition. The continuous spectra of hot stars are sensitive to $\mathrm{T}_{e}$ but insensitive to $g$ and to composition. Figure 2 shows some typical results, obtained by LUCY [14] for pure hydrogen stars with $\log g=4.0$. It is seen that for $\log \mathrm{T}_{e}$ in the range 4.1 to 4.5 the BALmer discontinuity, $\mathrm{D}=\log \left(\mathrm{F}_{3647}+\mathrm{F}_{\mathbf{3 6 4 7}}\right)$, is sensitive to $\mathrm{T}_{e}$, and that, for a fixed photo-visual magnitude, the ultra-violet fluxes are also sensitive to $T_{\iota}$. Figure 3 shows D as a function of spectral type plotted using the observations of CHALONGE and Divan [16]. It is seen that $D$ provides a very good criterion for determining the spectral type and for determining the effective temperatures of the models appropriate to each spectral type.
We consider the following models : an earlier model of UNDERHILL [17] for an 05 star ; recent

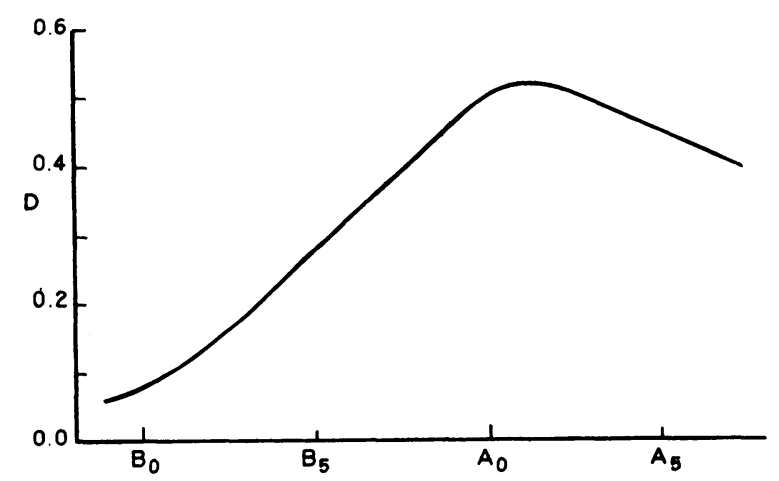

FIG. 3. - The Balmer discontinuity D as a function of spectral type [16]. 
UNDERHILL [7] models for early B stars ; models by Strom [18] for late B stars; a model by Osawa [19] for an A 3 star. These all have $\log g=4.0$, with the exception of the 05 model which has $\log g=4.2$. For the B star models we determine the spectral types using the measured values of D. Figure 4 shows $\log$ $\left(F_{1950} / F_{5560}\right)$ as calculated in this way and the observations of ALEXANDER, Bowen and HEDDLE [3] averaged for each spectral type. It is seen that the observed fluxes are less than those calculated by factors of about 2 or 3 , with some suggestion that the discrepancy is smaller at the ends of the range, 05 and $A$ 1. Figures 5 and 6 show similar results for $\lambda 1427$ and 1314, using the observations of CHUBB and BYram [2]. Here there is seen to be a discrepancy by a factor of about 3.5. A lucid discussion is given by STrömGREN [5], who considers a few selected stars for which accurate photometric measurements have been made in the visible. His results for $\lambda 1314$ are very similar to the results which we obtain from Figure 6 and his results concerning the measurements at $\lambda 2120$ [4] are similar to our results for $\lambda 1950[3]$.

In earlier discussions it was suggested that the models in the ultra-violet were in error by factors

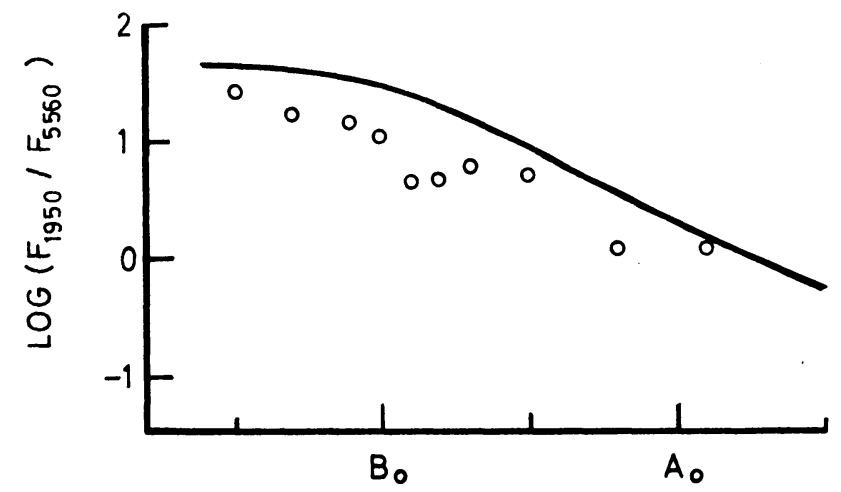

Fia. 4. - Observations [3] and model results for $\lambda 1950$.

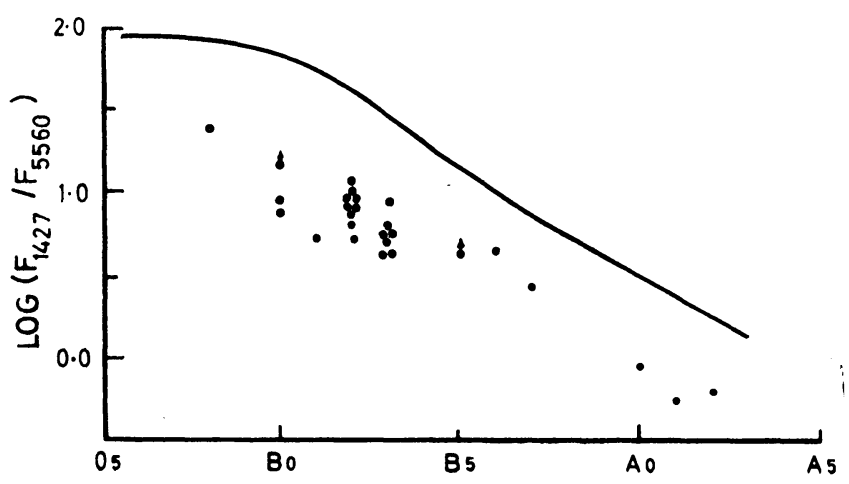

Fig. 5. - Observations [2] and model results for $\lambda 1427$.

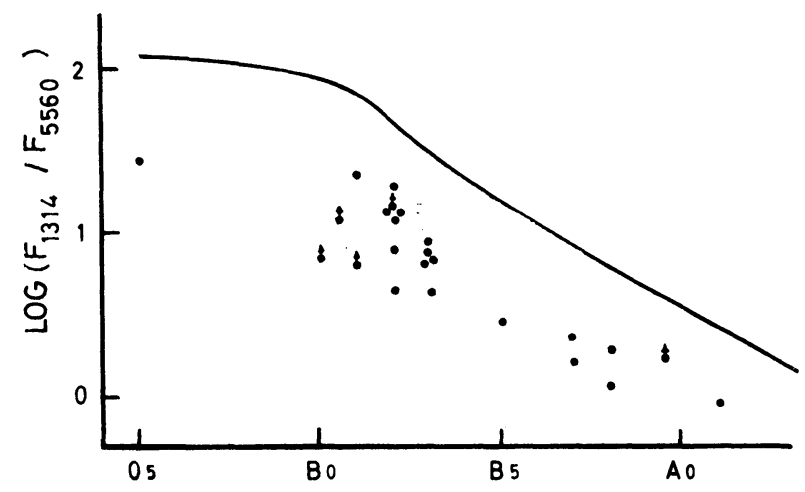

Fia. 6. - Observations [2] and model results for $\lambda 1314$.

of 20 [1] or even 30 [20]. It now seems fairly certain that the discrepancies are by factors of about 3 or 4 and rather less at the longer wavelength. $\lambda \simeq 2000 \AA$. It may be noted that, at each ultra-violet wavelength, the ratio of calculated flux to observed flux depends little, if at all, on spectral type, and that this would be consistent with the supposition that the discrepancies result from instrumental calibration. For the present discussion we shall assume that this is not the case and that the discrepancies shown in Figure 4,5 and 6 are real.

Most of the B stars which have been observed in the U. V. are generally assumed to be unreddened but one cannot be certain that interstellar absorption is completely negligible. HOYLE and WICKRAMASINGHE [21] consider a possible structure of interstellar grains which would give a very large ratio of $U$. V. to visible absorption and suggest that the entire discrepancy for the B star U. V. is due to interstellar absorption. The possibility of absorption by circumstellar grains has been discussed by Pecker [8], with particular reference to the abrupt intensity drop at $2400 \AA$ obtained by STEOHFR and MruIGaN [1]. After a careful discussion, STRöMaren concludes that it is " not too likely, but not impossible" that interstellar absorption is responsible for the the U. V. discrepancy. This question will, of course, be settled observationally as soon as we have U. V. observations for fainter stars which are definitely reddened. Results on the determination of interstellar absorption in the U. V. will be awaited with great interest.

We return to a discussion of the theoretical models. Although there may be some convective motions in B star atmosphere, they will not contribute sensibly to the energy transport or produce any significant deviations from hydrostatic equilibrium, and will therefore not be important for 
the calculation of continuum intensities. It also seems unlikely that allowance for non - LTE effects would cause any great change in the continuous spectrum but it may be noted that the continuous absorption is mainly due to excited states of hydrogen and hence that there is a strong coupling between non-LTE effects in the lines and in the continuum. The construction of nonLTE B star models should be a practical possibility in the near future.

The possibility of absorption by molecules, in stable or unstable states, has been proposed by STECHER and MrLIIGaN [1]. The main difficulty here is that for conditions in B star atmospheres the partition functions are such as to give a ratio of order $10^{-5}$ between the number of molecules and the number of atoms in states which contribute to the opacity. Absorption by unstable $\mathrm{H}_{2}$ is discussed further by SoLomon [12] and absorption by $\mathrm{HeH}+$ by WeRNER [13] ; it is concluded that these processes are not important. MEINEL [9] considers that B star atmospheres may be cooler than has been supposed but that there is an additional emission process involving formation of excited $\mathrm{H}_{2}$ which accounts for the maxima observed by StECHER and MrLIGAN at $\sim 2400 \AA$. It seems that this misses the main point of the problem, which is to reconcile the U. V. observations with observed features in the visible, such as the BaLmer discontinuity. In this connection it is of interest that a recent measurement of the angular radius of the A 0 star $\alpha$ LYRAE [22] enables one to obtain $\mathrm{H}_{\lambda}$, the flux at the stellar surface. The value obtained is in good agreement with that predicted by models which fit the spectral distribution in the visible [18].

Ultra-violet spectrum lines have been discussed by GAUSTAD and SPITZER [10] and by MoRToN [11] who calculates the line absorption within the pass bands of the CHUBB and Byram filters. At $1314 \AA$ he finds that line absorption may reduce the flux by a factor of $\sim 0.4$ for B 3 and B 6 , but that the lines are much less important for B 0 at at $1314 \AA$ and for all $B$ stars at $1427 \AA$. Unpublished calculations by Dr. E. TRefretz show that the accumulative effects of absorption by many weak lines are not large.

In addition to the direct effects of line absorption one must also consider blanketting, that is the effect of line absorption on the structure of the model. Approximate allowance for the Lyman lines may be made on shifting the effective wavelength of the Lyman limit [5], [23]. It is found that this does not produce any marked change in the continuous spectra. STrom [18] has constructed models which make allowance for the higher LYMAN and BALMER lines as opacity sources and, for $\alpha$ Lyrae, these give excellent agreement with observations in the visible, even in the region close to the BALMER limit. The ratio of U. V. to visible intensities is not, however, altered very much. Morton [11] finds that the total energy absorbed in the lines, including metal lines, is equal to about one third of the total flux. Further calculations of models with allowance for metal line opacities would be of interest.

We see that, although the discrepancies which we are discussing today are a good deal smaller than those which were discussed two or three years ago, the problem of the B-star U. V. is not yet satisfactorily resolved.

\section{STARS PROVIDED WITH PHOTON COUNTERS.}

It is generally accepted that, due to absorption by interstellar hydrogen, stellar observations cannot be made in the region between the Lyman limit and the soft $\mathrm{X}$-rays: This overlooks the fact that certain very hot stars are provided with their own photon counters, in the form of planetary nebulae, and that, as ZANsTRA remarks [24], this enables us to do space research at low cost.

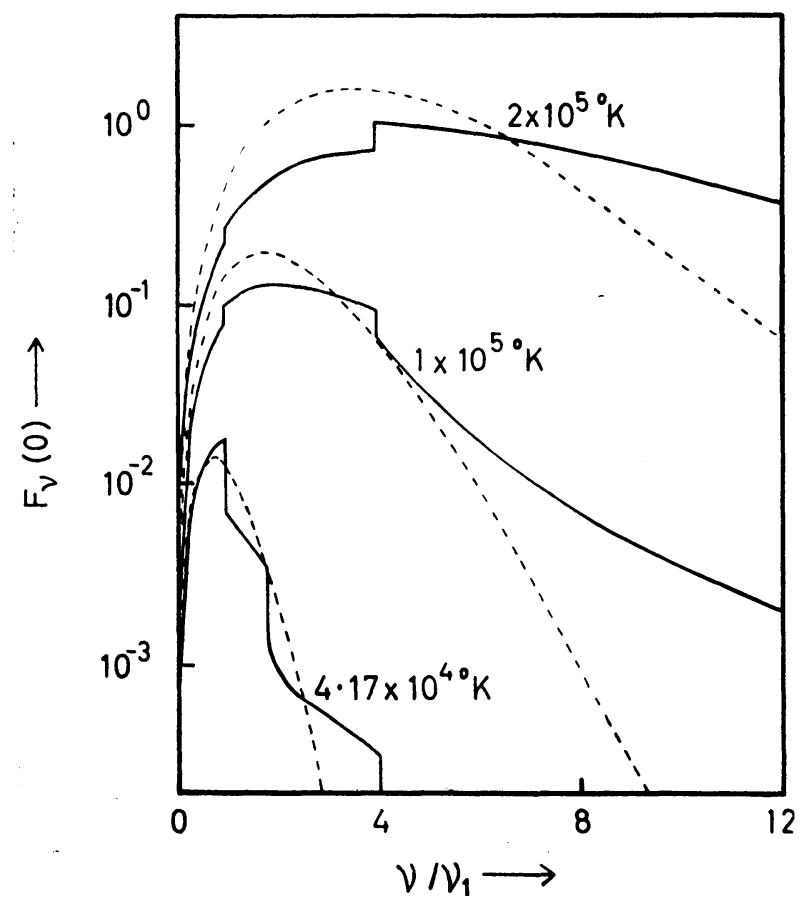

Fia. 7. - Calculated fluxes for central stars of planetary nebulae [15]. $F_{v}(0)$ is the flux in ergs $\mathrm{cm}^{-2} \mathrm{~g}^{-1}$ per unit frequency at the surface of the star, $v_{1}$ is the frequency of the Lyman limit. 
Model atmospheres for very hot stars have been calculated by Mrs. GrbBIE [15], [25]. Figure 7 shows the calculated flux distributions. It is seen that the H I and He II Lyman discontinuities may appear in absorption or in emission, depending on the values of $T_{e}$ and of $g$. It is
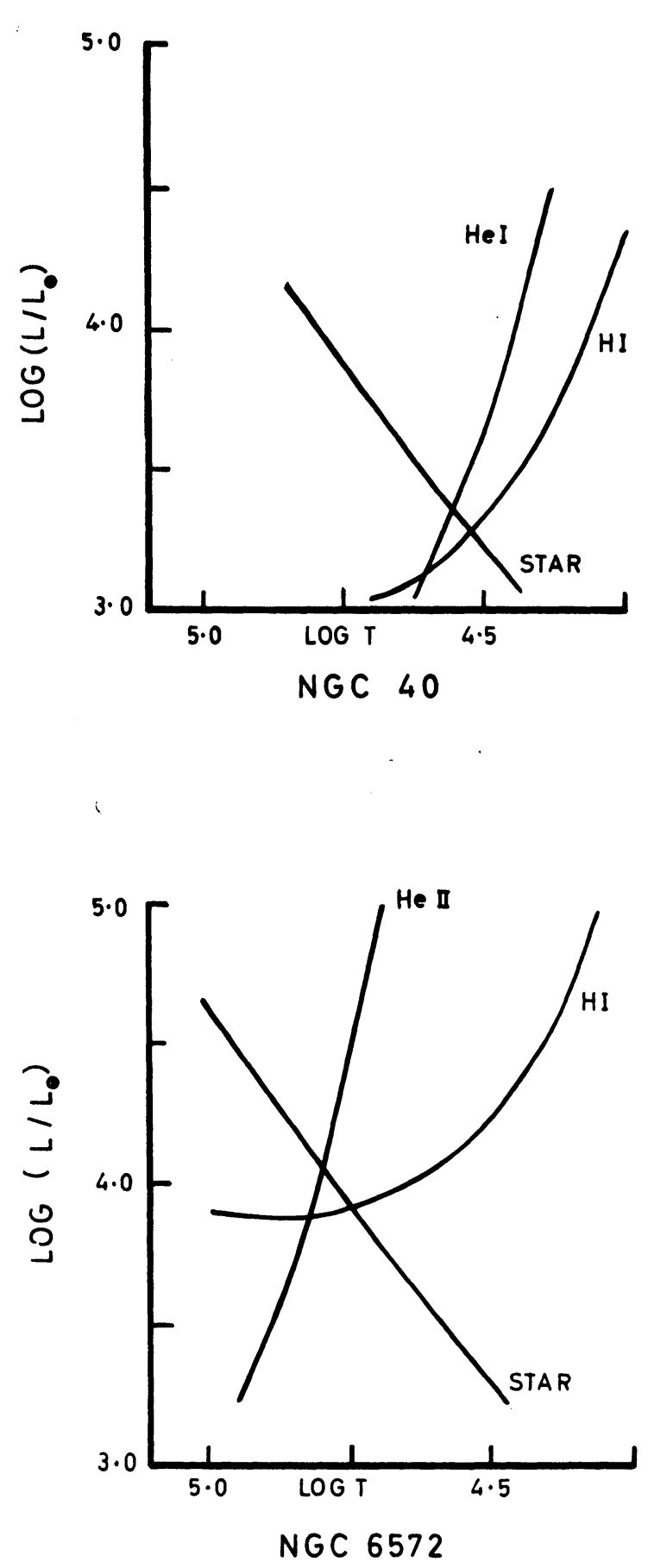

also seen that the flux distributions are not markedly different from black body fluxes.

We have observations of these stars in the visible and, from observations of the nebular quantum counters, we can deduce the numbers of quanta beyond the H I, He I and He II limits.
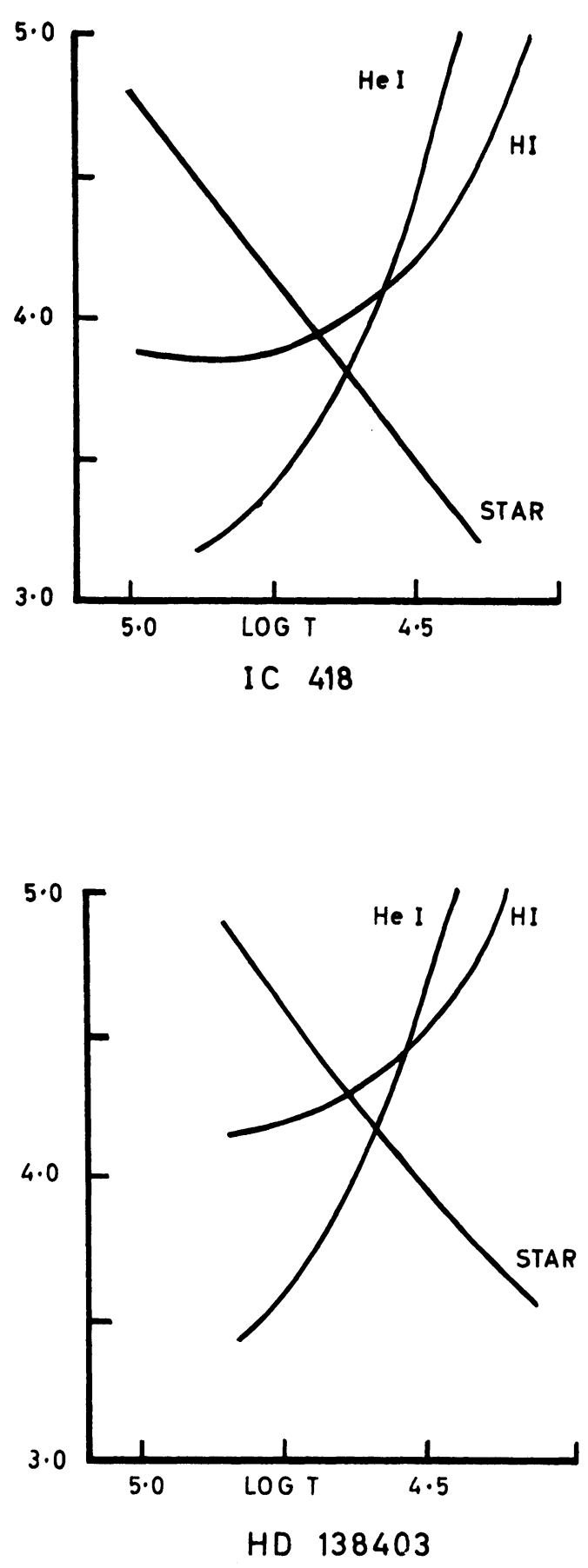

Fia. 8. - Luminosity-temperature plots for central stars of planetary nebulae. The curves STAR are from observations in the visible and the curves $\mathrm{H} I, \mathrm{He} I$ and $\mathrm{He}$ II are for numbers of quanta beyond the various limits, deduced from intensities of nebular lines. For these plots, the distance scale of $\mathrm{O}^{\prime}$ DeLL [35] is adopted. 
It is, however, necessary to be sure that the counters are $100 \%$ efficient [26], [27]. In Figure 8 we consider one nebula for which the $\mathrm{H} \mathrm{I}$ and $\mathrm{He}$ II counters are $100 \%$ efficient and three nebulae for which the $\mathrm{HI}$ and $\mathrm{He} \mathrm{I}$ counters are $100 \%$ efficient. Each observation gives a line in a plot of $\log \left(\mathrm{L} / \mathrm{L}_{\odot}\right)$ against $\log \mathrm{T}_{e}$, the stars being assumed to radiate as black bodies. It is seen that good consistency is obtained. The implications of these results are discussed further elsewhere [27].

\section{Nebulat.}

Reported nebular glows in the band 1225 - $1350 \AA$ have not been confirmed in more recent work [28].

Expected ultra-violet lines in the spectra of gaseous nebulae have been discussed by CoDe [29], ALLER [39] and OsTERBRock [31]. In high excitation planetaries an important source of electron kinetic energy is provided by ionization of $\mathrm{H}$ by $\mathrm{He}$ II Ly $\alpha$ quanta and it is suggested by HuMmer and SEATON [32] that a good part of this energy may be used in excitation of resonance lines such as C IV $2 s-2 p, \lambda$ 1551, 1548. For bright high excitation planetaries the flux in the C IV lines may be as high as $\sim 10^{-8}$ ergs $\mathrm{cm}^{-2} \mathrm{~s}^{-1}$ at the earth if interstellar absorption is not important but will generally be reduced by one or two orders of magnitude by absorption.

\section{INTERstellar MATter.}

We have already mentioned the importance of determining the law of interstellar reddening for U. V. wavelengths. Problems of absorption and scattering by the interstellar gas have been discussed by SPITZER and ZABRISKIE [33] and by Cook [34]. It is shown by Cook that the profiles for scattering of Lyman line radiation by interstellar hydrogen can be deduced from $21 \mathrm{~cm}$ observations. A similar theory is applied to absorption by molecular hydrogen and it is shown that the amount of interstellar $\mathrm{H}_{2}$ might be determined from observations on $\mathrm{O}$ and $\mathrm{B}$ stars at about $1100 \AA$.

\section{AOKNOWLEDGEMENTS}

I have benefited from discussions with a number of my colleagues, in particular Dr. E. TremFtr, Dr. G. Peach, Mrs. K. B. Gebbie and Dr. D. W. O. HeddLE.

Manuscrit reçu le 23 septembre 1964.

\section{REFERENCES}

[1] Stegher T. P. and Milligan J. E., 1962, Ap. J., 136, 1 .

[2] ChUBB T. A. and ByramE.T., 1963, Ap.J.,138,617.

[3] Alexander J. D. H., Bowen P. J. and Heddle D. W. O., 1964, Proc. Roy. Soc., A279, 510.

[4] Golledae I. S. and Packer D. M., 1963, A. J., 68, 537.

[5] Strömaren B., 1964, Rev. Mod. Phys., 36, 532.

[6] Canavageia R. and Peoker J.-C., 1953, Ann. d'Astr., 16, 47.

[7] UNDERHIIL A. B., 1963, Space. Sci. Rev., 1, 749.

[8] Phoker J.-C., 1963, Space Sci. Rev., 1, 729.

[9] Metnel A. B., 1963, Ap. J., 137, 321.

[10] GaUstad J. E. and Spitzer L., 1961, Ap. J., 134, 771.

[11] Morton D. C., 1964, Ap. J., 139, 1383.

[12] Solomon P. M., 1964, Ap. J., 139, 999.

[13] Werner M. W., 1964, Ap. J., 139, 1014.

[14] LUOY L., 1963, Thesis, Manchester.

[15] GrbBie K. B., 1965, Thesis, London.

[16] Chalonge D. and Divan L., 1952, Ann. d'Astr., 15, 201.

[17] Underminl A. B., 1951, Publ. Dom. Astrophys. Obs., 8, 357.

[18] Strom S. E., 1964, Thesis, Harvard.

[19] OsAWA K., 1956, Ap. J., 123, 513.
[20] Bogatss A. E., 1961, Mém. Soc. Roy. Sci. Liège, 4,459 .

[21] Hoyle F. and Wiokramasinghe N. C., 1963, $M . N ., 126,401$.

[22] Hanbury Brown R., Hazard C., Davies J. and ALlen L. R., 1964, Nature, 201, 111.

[23] Cayrel R., 1957, Thèse, Paris.

[24] Zanstra H., 1960, B. A. N., 15, 237 ; Quarterly $J ., R . A . S ., 2,137$.

[25] Gebie K. B. and Seaton M. J., 1963, Nature, 199, 580.

[26] Staton M. J., 1960, Rep. Progr. Phys., 23, 313.

[27] Harman R. J. and Seaton M. J., [1964, Ap. J., $140,824$.

[28] Byram E. T., Chubb T. A. and Friedman H., $1964, A p . J ., 139,1135$.

[29] Code A. D., 1960, A. J., 65, 278.

[30] Aller L. H., 1961, Mém. Soc. Roy. Sci. Liège, 4,535

[31] Osterbrook D. E., 1963, Plan. Sp. Sci., 11, 621

[32] Hummer D. G. and Seaton M. J., 1964, M. N., 127, 217.

[33] SPITZER L. and ZABRISKIE F. R., 1959, P. A.'S. P. $71,412$.

[34] Cook A. H., 1963, Quarterly J., R. A. S., 4, 203

[35] O'D fll C. R., 1962, Ap. J., 135, 371. 